CORRECTION

https://doi.org/10.1038/s41586-019-1106-6

\title{
Author Correction: Spatially resolved steady-state negative capacitance
}

Ajay K. Yadav, Kayla X. Nguyen, Zijian Hong,

Pablo García-Fernández, Pablo Aguado-Puente,

Christopher T. Nelson, Sujit Das, Bhagwati Prasad,

Daewoong Kwon, Suraj Cheema, Asif I. Khan, Chenming Hu, Jorge Íñiguez, Javier Junquera, Long-Qing Chen,

David A. Muller, Ramamoorthy Ramesh \& Sayeef Salahuddin

Correction to: Nature https://doi.org/10.1038/s41586-018-0855-y, published online 14 January 2019.

In this Letter, the first name of author Bhagwati Prasad was misspelled

Bhagawati. This error has been corrected online. 